\title{
Closure of salient regions determines search for a collinear target configuration
}

\author{
Markus Conci and Hermann J. Müller \\ Ludwig-Maximilians Universität, Munich, Germany \\ AND \\ Mark A. Elliott \\ Ludwig-Maximilians Universität, Munich, Germany \\ and National University of Ireland, Galway, Ireland
}

\begin{abstract}
Grouping operations offer an effective mechanism by which to structure complex visual scenes. Among the various principles that support element integration, closure may be regarded as a main factor in shape extraction. In five experiments, we investigated the impact of grouping by closure on search for target configurations made up of collinearly arranged corner junctions. Systematic variations in the configuration of distractor elements were explored to investigate how the figural information of distractors interferes with target detection. The results showed no search interference for distractor configurations that were open forms. By contrast, distractors making up closed forms reduced the efficiency of search performance, indicating closure as a major contributor to form detection in multielement configurations. In conclusion, the effects reported may be considered to arise from mechanisms of unit formation that support the rapid extraction of salient regions to guide search.
\end{abstract}

Unit formation can be regarded to be a major function in the organization of perceptual information. Gestalt psychology (Koffka, 1935; Wertheimer, 1923) originally demonstrated that the association of distinct elements into groups is subject to a set of basic principles (e.g., similarity, closure, and proximity), which are regarded as primary operations forming perceptual units prior to detailed analysis. In contrast, constructivist approaches assume that visual perception proceeds from the analysis of elementary features and progresses by the integration of features into coherent objects, which involves visual attention (Treisman \& Gelade, 1980). Psychophysical investigations in support of a constructivist position have identified a number of visual primitives, organized in terms of separable dimensions of such basic features (e.g., luminance, color, or orientation; see Treisman \& Gormican, 1988; Wolfe, 1998; Wolfe \& Horowitz, 2004).

Besides basic primitives, visual processing of more complex units has been documented, with unit formation operating in an effortless manner. Support for this idea is provided by evidence that perceptual configurations may be detected more efficiently than their isolated features (Pomerantz, Sager, \& Stoever, 1977; see also Treisman $\&$ Paterson, 1984). In addition, visual search studies have shown that component parts may be grouped prior to the engagement of attention (e.g., Moore \& Egeth, 1997; Rensink \& Enns, 1995), and that search may be more effectively guided by integrated shapes than by corresponding local features (Found \& Müller, 1997). Moreover, there is evidence that early visual, or preattentive, processes operate on the basis of a variety of grouping principlesnotably, similarity (Duncan, 1984; Duncan \& Humphreys, 1989; Humphreys, Quinlan, \& Riddoch, 1989), closure (Elder \& Zucker, 1993; Han, Humphreys, \& Chen, 1999a; Kovács \& Julesz, 1993), and proximity (Han, Humphreys, $\&$ Chen, 1999b). These studies complement those in which the primitive features have been described by showing that complex visual units are, at least to some extent, available at early stages of processing.

In general, models of perceptual grouping assume that element integration is achieved on the basis of similarity and proximity, segregating distinct regions within the visual field (e.g., Geisler \& Super, 2000). Moreover, collinearity and closure supply critical information for shape extraction, supporting the separation of figure from ground (e.g., Elder \& Zucker, 1993; Field, Hayes, \& Hess, 1993; Kovács \& Julesz, 1993). Visual search studies have demonstrated that the integration of separate collinear vertices into closed forms permits efficient processing, whereas groupings that do not exhibit closure are processed relatively inefficiently (Donnelly, Humphreys, \& Riddoch, 1991; Donnelly, Weekes, Humphreys, \& Albon, 1998). For instance, search for a misoriented vertex element was performed efficiently and independently of the number of distractor vertices when the distractor elements could be grouped on the basis of collinearity and closure to form a 
coherent shape description. In contrast, when the distractor vertices could not be integrated on this basis, search was slow, with reaction time (RT) increasing as a function of the number of distractor junctions.

Basic grouping operations are conceptualized as processes that operate on separate elements with the aim of integrating component parts into coherent wholes. In addition, certain configurations may be interpreted by the visual system in such a way that the resulting unit does not simply represent the compound of related elements linked together by Gestalt operations but defines a qualitatively different illusory figure. Figure 1 contrasts examples of such an illusory figure (Figure 1A; Kanizsa, 1955) with a corresponding grouping that integrates separate elements on the basis of collinearity and closure only (Figure 1B). Whereas Figure 1A appears as a bright central square exhibiting sharp boundaries, Figure 1B does not, in general, support a corresponding interpretation. ${ }^{1}$

Studies in which visual search for illusory figures has been investigated have thus far led to equivocal interpretations with regard to the stage at which illusory figures are completed. Whereas some authors have proposed that search is based on preattentive completion of illusory contours (Davis \& Driver, 1994), others have argued that there is no definite evidence to support the idea that Kanizsa-type figures are detected in parallel (Grabowecky \& Treisman, 1989; Gurnsey, Poirier, \& Gascon, 1996). Follow-up investigations (Conci, Müller, \& Elliott, 2007) have shown that surface specifications in distractors influence search for an illusory target figure, whereas contour specifications have little direct effect on target detection. These conflicting findings and viewpoints suggest that only certain figural attributes are coded for search. The specification of surface information may be such an attribute in visual search for a Kanizsa figure (Figure 1A). However, basic principles of grouping may be equally effective in providing information to guide search, without involving the formation of an illusory figure. For example, specifications of collinearity and closure may supply information supporting efficient detection of the (target) figure illustrated in Figure 1B.

To examine whether closure influences target detection, a series of visual search experiments were conducted. Observers had to report, as rapidly and accurately as possible, the presence or absence of a closed target square (as illustrated in Figure 1B) among different types of distractor configurations composed of similar corner junctions.

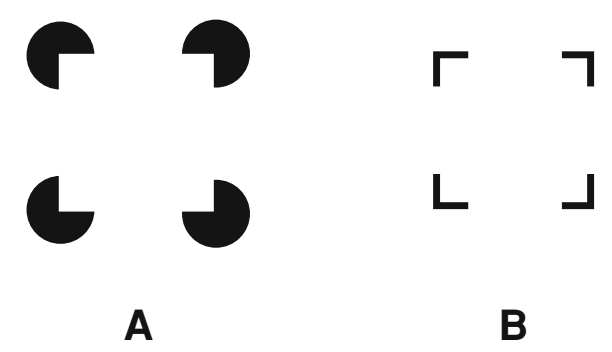

Figure 1. (A) An example of a Kanizsa square inducing an illusory figure. (B) A corresponding configuration composed of corner junctions without comparable figural emergence.
The distractor configurations were systematically varied in the degree to which closure could serve as a grouping cue. This permitted exploration of whether systematic variation of distractor closure would lead to interference in search for a target configuration exhibiting closure (cf. Duncan \& Humphreys, 1989).

In Experiment 1, we compared the efficiency of detecting a target square formed by four collinearly organized junctions among similarly organized open- and closedform distractors (see Figure 2A for the two distractor types and Figure 2B for an example search display). The results revealed that only closed-form, but not open-form, distractors, produced systematic interference (in terms of a slowing of search rates), suggesting that closure influences search efficiency. In Experiment 2, we examined the effect of reducing the physical specification of the junction elements, thereby weakening the degree of closure afforded by collinear junctions (see Figure $2 \mathrm{C}$ for an example search display). The reduction in element specification produced a general slowing of the search rates, which was, however, more marked with closed- than with open-form distractors. In Experiment 3, we introduced the reverse manipulation: presentation of additional perpendicular lines that terminated adjacent to the continua formed between collinear junctions, potentially enhancing closure (see Figure 5). As a result, both open- and closed-form distractors were found to interfere with target detection - with larger interference, however, for closed- than for open-form distractors. These findings again point toward a specific role for closure in search. Experiment 4 was conducted to rule out the possibility that the effects observed in Experiment 1 were simply due to differences in overall size between the target and the distractor configurations. Despite controlling for size (by standardizing the outline dimensions of the target and distractor configurations; see Figure 7A-II), Experiment 4 revealed a pattern of interference effects nearly identical to that in Experiment 1. This supports an interpretation of this pattern in terms of closure, rather than outline size, of the distractor configurations. Finally, Experiment 5 was carried out to examine whether or not the effects attributed to closure may be better explained in terms of the similarity between the junction elements making up the target and distractor configurations, with similarity defined in terms of the number of matching element orientations. To test this assumption, in Experiment 5, we examined whether performance differences would be obtained when we varied closure while controlling for similarity. In agreement with Experiments 1-4, detection RTs were longer overall and increased more markedly with display size when closure was not a defining attribute of the target. In sum, all five experiments revealed consistent evidence for closure's exerting a major influence on search for target configurations composed of corner junctions, with search efficiency depending on physical form specification and the number of candidate closed forms in the visual display.

\section{EXPERIMENT 1}

Experiment 1 was designed to examine whether closure influences search for a collinear target configuration 
A
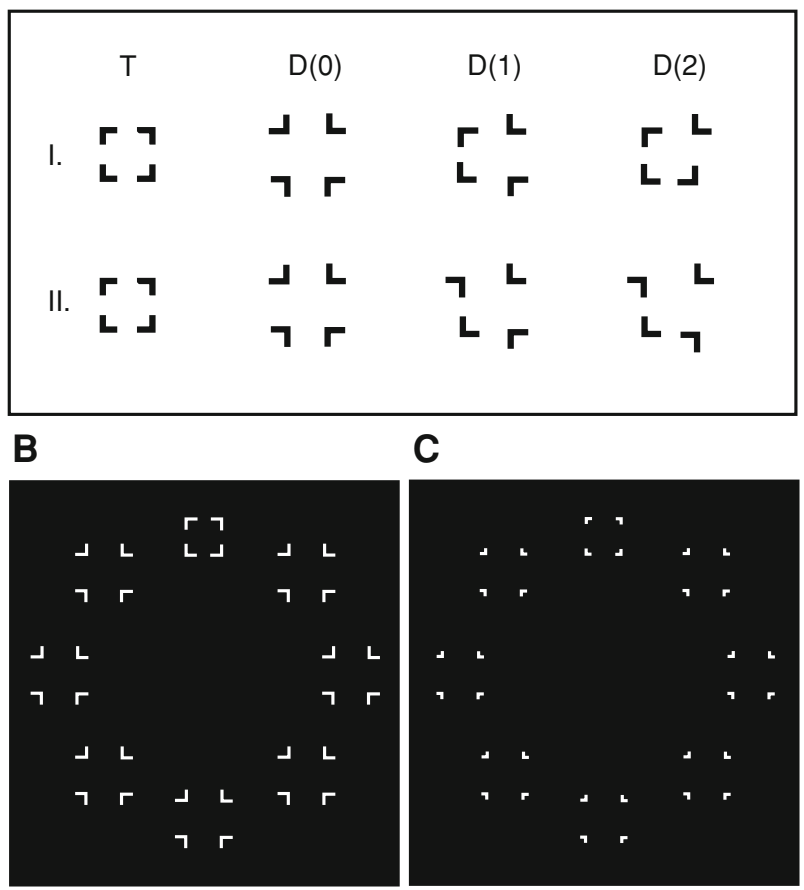

Figure 2. (A) Examples of target (T) and distractor (D) configurations presented in Experiment 1. Distractors could contain zero, one, or two collinear continuations $[\mathrm{D}(0)-\mathrm{D}(2)]$ between adjacent corner junctions that promoted either a closed form (I) or a corresponding open form (II). (B) and (C) Examples of targetpresent displays with eight candidate groupings in Experiments 1 and 2, respectively (with the physical specification of the junction line segments reduced by $\mathbf{5 0 \%}$ in Experiment 2).

composed of four junction elements arranged to form the corners of a square. The target was presented within search displays that contained distractor configurations composed of similar corner junctions, which were, however, arranged so that they produced either a partially closed form or a corresponding open form (see Figures 2A-I and 2A-II for examples). For both types of distractors, the number of collinear junctions increased from zero (common baseline) through one to two [in Figure 2A, the conditions are referred to as $\mathrm{D}(0), \mathrm{D}(1)$, and $\mathrm{D}(2)$, respectively]. The two types of distractor configurations differed in that either both of the neighboring collinear junctions were oriented toward the inside of the configuration (closed form) or one was oriented inward and the other outward (open form). This manipulation permitted establishing whether a gradual increase of closure would influence (i.e., interfere with) detection of a closed-form target.

\section{Method}

Participants. Eight observers ( 1 of them male, mean age $=25.5$ years) with normal or corrected-to-normal visual acuity participated in the experiment, receiving payment of $€ 8$ per hour.

Stimuli. The stimuli were generated by an IBM-compatible PC and were presented in white $\left(1.83 \mathrm{~cd} / \mathrm{m}^{2}\right)$ against a black $\left(0.02 \mathrm{~cd} / \mathrm{m}^{2}\right)$ background at eight possible locations on a 17 -in. monitor screen. The stimulus configurations were placed on a virtual circle around the screen center, with a radius of $8.75^{\circ}$ of visual angle (at a viewing distance of $55 \mathrm{~cm}$ ). An example display with eight stimulus configurations is shown in Figure 2B. Each configuration, or candidate grouping, was composed of four $90^{\circ}$ corner junctions (with junction line segment $1^{\circ}$ in length) arranged in a square-like form. As is depicted in Figure 2A, the target (T) was defined by the collinear arrangement of all four corner junctions (subtending $2.9^{\circ} \times 2.9^{\circ}$ of visual angle). In contrast, distractor configurations (D) were produced by rotating junction elements so that each grouping contained only zero, one, or two aligned junction-junction continua (subtending up to $4.9^{\circ} \times 4.9^{\circ}$ when all the junction convexities pointed toward the center of the configuration; see Figure 2A for examples). In this way, two types of distractor configurations were generated: partially closed and partially open groupings (for examples, see Figures 2A-I and 2A-II, respectively). Trial displays could contain one, two, four, or eight candidate groupings (the display size), with each grouping presented in a random orthogonal orientation. On $50 \%$ of the trials, a target configuration was present in the display. For displays with fewer than eight candidate groupings, the stimulus positions were chosen pseudorandomly from among the eight possible locations, with the following constraints: For display sizes of two, candidate groupings were presented at diametrically opposite positions only, and for display sizes of four, groupings were presented at every second position of the eight possible locations.

Procedure. Each trial started with the presentation of a central fixation cross for $500 \mathrm{msec}$. The fixation cross was then immediately replaced by the search display, to which the observers responded with a speeded target-absent/-present response via mouse keys. Displays remained on-screen until a response was recorded. In the case of an erroneous response or a time-out (i.e., after a period of 2,500 msec without reaction), feedback was given by a computergenerated tone, and an alerting message was presented for $500 \mathrm{msec}$ at the center of the screen. Each trial was separated from the next by an interval of $500 \mathrm{msec}$

The experiment was conducted over two sessions, each presenting either open-form or closed-form distractors. Each session consisted of 12 blocks of 80 trials, with the number of collinear continuations $[\mathrm{D}(0), \mathrm{D}(1)$, and $\mathrm{D}(2)]$ constant throughout a block. Collinear continuation blocks were administered in pseudorandom order on an observer-by-observer basis. In summary, the independent variables were target (T; present or absent), display size (DS; 1, 2, 4, or 8 configurations), distractor type (DT; open or closed form), and collinear continuations in distractor configurations $(\mathrm{CC} ; 0,1$, or 2 continuations), with 40 trials per condition.

\section{Results}

RT analysis. RTs on trials on which a response error was made $(2.3 \%)$ were removed from the data set prior to RT analysis. Figure 3 presents the mean correct RTs and the proportions of errors as a function of display size, separately for the open- and closed-form distractors (see figure columns $\mathrm{A}$ and $\mathrm{B}$, respectively) and the various collinear continuation (i.e., CC) conditions.

The whole data set was initially examined by a repeated measures ANOVA with the factors of distractor type, collinear continuations, target, and display size. This ANOVA revealed that all the main effects and interactions, including the four-way interaction $[F(6,280)=13.13, p<$ $.001]$, were significant. Next, to decompose the four-way interaction, the data sets for open- and closed-form distractor types were analyzed separately by two ANOVAs with the factors of collinear continuations, target, and display size. For the open-form distractors, the ANOVA revealed that the main effects for target and display size and their interaction were significant $[\mathrm{T}, F(1,7)=20.74, p<$ $.01 ; \mathrm{DS}, F(3,21)=10.76, p<.001 ; \mathrm{T} \times \mathrm{DS}, F(3,119)=$ 
A
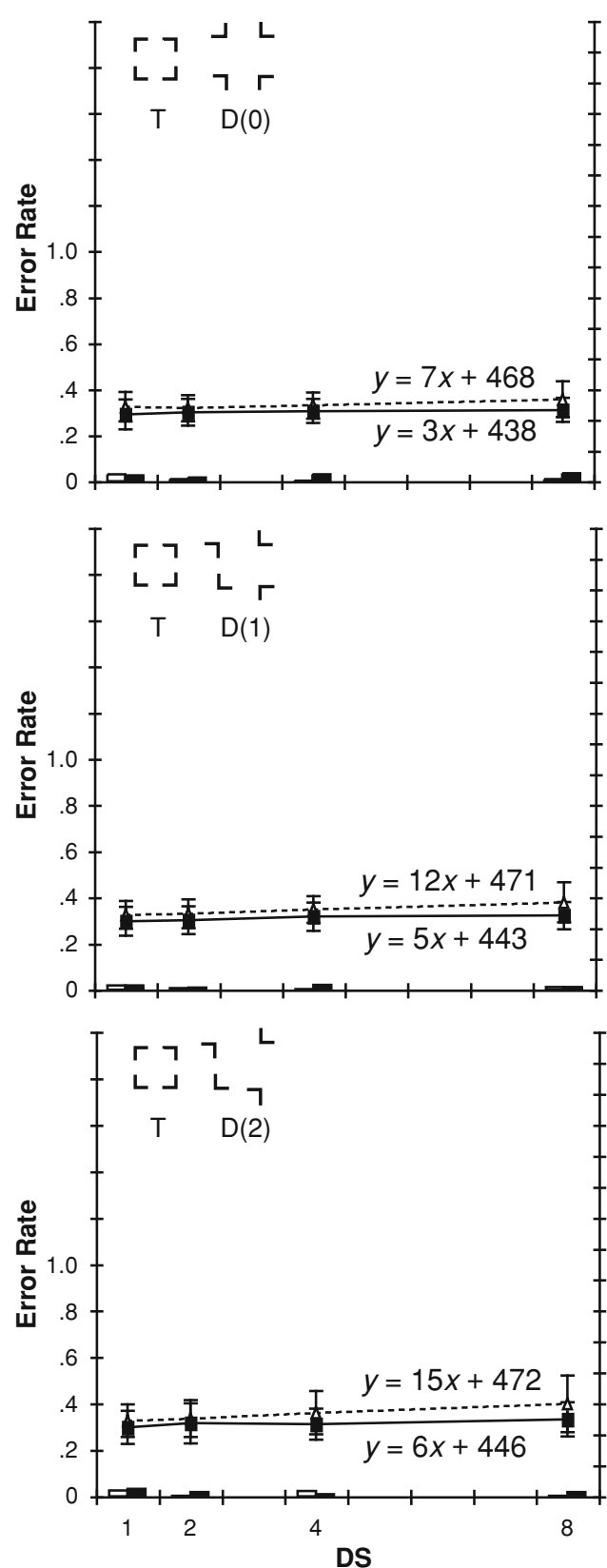

B

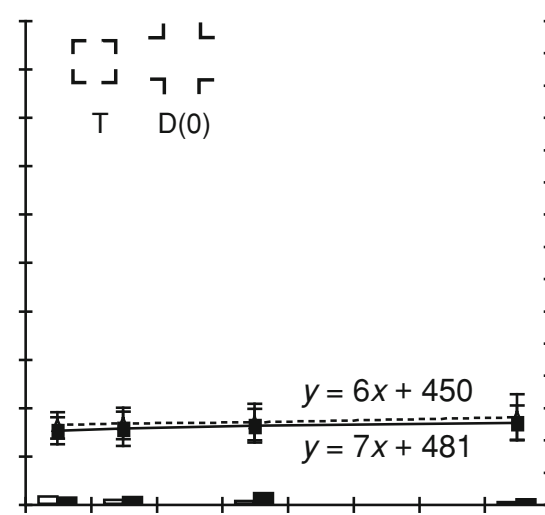

3,000
2,800
2,600
2,400
2,200
2,000
1,800
1,600
1,400
1,200
1,000
800
600
400
200
0
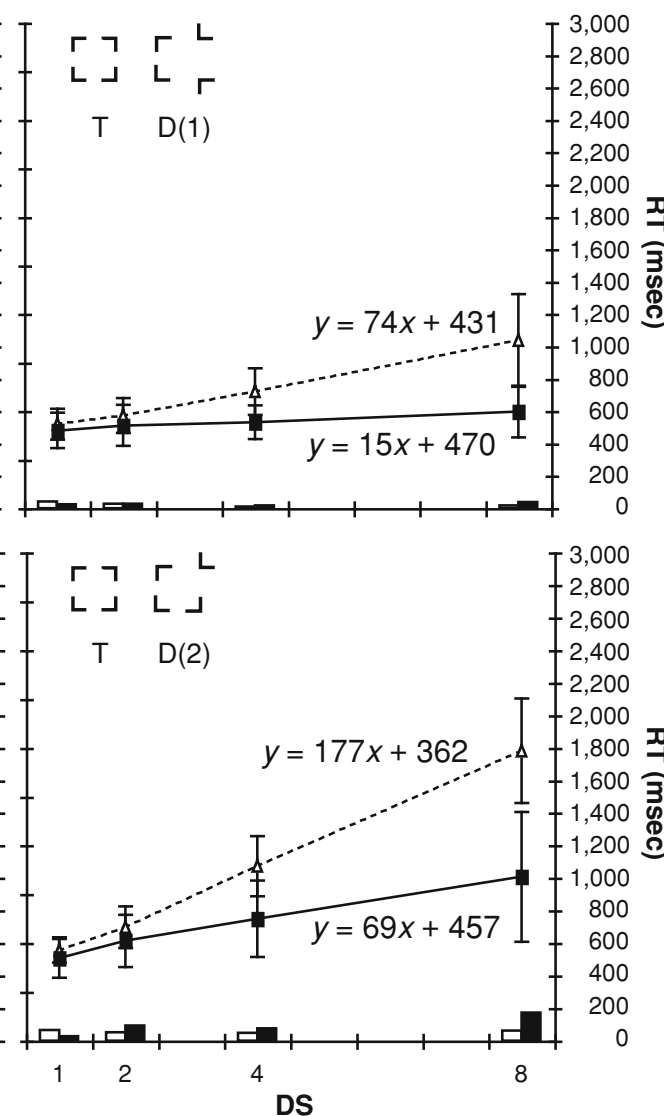

Figure 3. Mean reaction times (RTs, with associated $S D$ s) and error rates in Experiment 1 as a function of display size (DS), separately for open-form (A, left column) and closed-form (B, right column) distractors with zero, one, and two collinear continuations (top, middle, and bottom graphs, respectively). Each graph shows the prototype target ( $T$ ) and an example of a distractor (D) and plots RTs and error rates separately for target-absent (dotted line, white bars) and target-present (solid line, black bars) trials. In addition, the function for the best-fitting straight line is given for each search RT function, with the search rate and base $\mathrm{RT}$ estimates.

$6.39, p<.001]$. Target-present RTs were overall shorter than target-absent RTs, and RTs increased as a function of display size, with a somewhat more marked increase for target-absent than for target-present RTs (DS effects of 11.33 vs. $4.67 \mathrm{msec} /$ item). However, despite the significant display size effects, the search rates were within a range taken to be indicative of efficient, parallel search, independently of the number of collinear continuations in the distractor configurations.

By contrast, for the closed-form distractors, the ANOVA revealed that all the effects were significant $[\mathrm{T}, F(1,7)=$ $72.19, p<.001 ; \mathrm{DS}, F(3,21)=97.61, p<.001 ; \mathrm{CC}$, $F(2,14)=274.81, p<.001 ; \mathrm{T} \times \mathrm{DS}, F(3,119)=64.79$, $p<.001 ; \mathrm{T} \times \mathrm{CC}, F(2,119)=55.49, p<.001 ; \mathrm{DS} \times \mathrm{CC}$, 
$F(6,119)=97.28, p<.001 ; \mathrm{T} \times \mathrm{DS} \times \mathrm{CC}, F(6,119)=$ 20.11, $p<.001$ ]. As can be seen from Figure 3B, RTs increased markedly with increasing display size as the number of collinear continuations in the distractor configurations increased, with search rates (target absent [target present]) decreasing from 7 [6] through 15 [74] to 69 [177] msec/item with zero, one, and two continuations, respectively. Thus, the significant four-way ANOVA in the overall ANOVA is accounted for by the fact that this pattern of increasing interference, associated with an increasing number of collinear continuations in the distractor configurations, was manifest only for closed-form, but not for open-form, distractor types.

Error analysis. RTs on trials on which a response error was made tended to be longer overall than correct RTs, which argues against RT performance's being contaminated by speed-accuracy trade-offs (this was the case in all the subsequent experiments as well).

Overall, erroneous responses were relatively rare ( $2.3 \%$ of all the trials; $2.8 \%$ misses, $1.7 \%$ false alarms). The arcsine-transformed error rates were examined by ANOVAs of the same design as those applied to the RT data. The overall ANOVA failed to reveal the four-way interaction to be significant. For the open-form distractors, the ANOVA revealed the target and display size main effects to be significant $[\mathrm{T}, F(1,7)=6.83, p<.05$; $\mathrm{DS}, F(3,21)=5.66, p<.01]$. Error rates increased with display size, and target misses (target-absent trials) were more frequent than false alarms (target-present trials), despite the fact, mentioned above, that no speed-accuracy trade-offs were evident.

Similarly, for the closed-form distractors, the ANOVA revealed target misses were more common than false alarms $[\mathrm{T}, F(1,7)=8.11, p<.05]$, with a significant rise in miss rates with increasing display size $[\mathrm{T} \times \mathrm{DS}$, $F(3,119)=5.77, p<.01]$. Furthermore, the error ratesin particular, those for larger display sizes - were affected by the number of collinear continuations [CC, $F(2,14)=$ $14.05, p<.001 ; \mathrm{CC} \times \mathrm{DS}, F(6,119)=4.09, p<.001]$. Thus, in the closed-form condition, increasing the number of collinear continuations in the distractors produced increasing interference with search performance in terms not only of response speed, but also of response accuracy. This pattern is in contrast with performance in the openform condition, in which performance was unaffected by the number of collinear continuations in the distractors.

\section{Discussion}

Experiment 1 revealed that the efficiency of target detection was unaffected by the presence of open-form distractors in the display: Search was efficient (parallel) in all the conditions (across conditions, the search rates averaged 4.67 [11.33] msec/item), and the number of collinear continuations between junctions had no effect on performance. In contrast, closed-form distractors were revealed to significantly influence performance, with a systematic slowing of the search rates when the number of collinear continuations between corner junctions increased from zero through one to two. Each additional collinear continuation in distractors slowed the search time per item by 31.0 [85.5] msec, as compared with 1.5 [4.0] msec with open-form distractors. As a result, with one and two collinear continuations, the search rates (15 [74] and 69 [177] msec/item, respectively) were outside the range taken to be indicative of efficient search.

In summary, Experiment 1 revealed a search asymmetry between open- and closed-form distractors, so that only closed-form distractors interfere with target detection, with the degree of interference dependent on the number of collinear continuations. This pattern suggests that although closed- and open-form distractors share identical collinear boundaries, interference is observed only when distractors exhibit closure.

\section{EXPERIMENT 2}

Experiment 1 provided evidence that closure in distractor configurations reduces the efficiency of detecting a target figure defined by closure. In Experiment 2, this influence was examined further by reducing the size of the corner junctions, which was expected to reduce the potential effects of closure for both closed- and open-form distractors. This manipulation was premised on the finding that the strength of grouping between collinear elements varies in relation to the ratio of physically specified to total edge length (Shipley \& Kellman, 1992). Experiment 2 was, in almost all respects, identical to Experiment 1, except that corner junction elements were reduced in size by $50 \%$, relative to those in Experiment 1, without altering the overall size of the configurations. As in Experiment 1, the efficiency of closed-form detection was compared for open- and closed-form stimulus configurations.

\section{Method}

At a viewing distance of $55 \mathrm{~cm}$, the line segments making up a corner junction in target and distractor configurations were $0.5^{\circ}$ in length, as compared with $1^{\circ}$ in Experiment 1 (see Figure $2 \mathrm{C}$ for an example). Eight observers ( 3 of them male, mean age $=24.6$ years) with normal or corrected-to-normal vision participated in Experiment 2. All other methodological details were the same as those in Experiment 1.

\section{Results}

RT analysis. As in Experiment 1, trials on which a response error was made $(4.1 \%)$ were removed from the data set prior to RT analysis. Figure 4 presents the mean correct RTs and the proportions of errors as a function of display size, separately for the open- and closed-form distractors (see figure columns A and B, respectively) and for the various collinear continuation conditions. As can be seen, with the reduced-size junction elements presented in Experiment 2, search was less efficient overall, with slowed search rates in all the conditions, relative to those in Experiment 1. However, the detrimental effect of the reduced element specification was more marked with closed-form than with open-form distractors.

The whole data set was initially examined by an ANOVA with the factors of distractor type, collinear continuations, target, and display size. This ANOVA revealed that all the main effects and all the two- and three-way interactions (except distractor type $\times$ target $\times$ collinear continuations) 
A

B

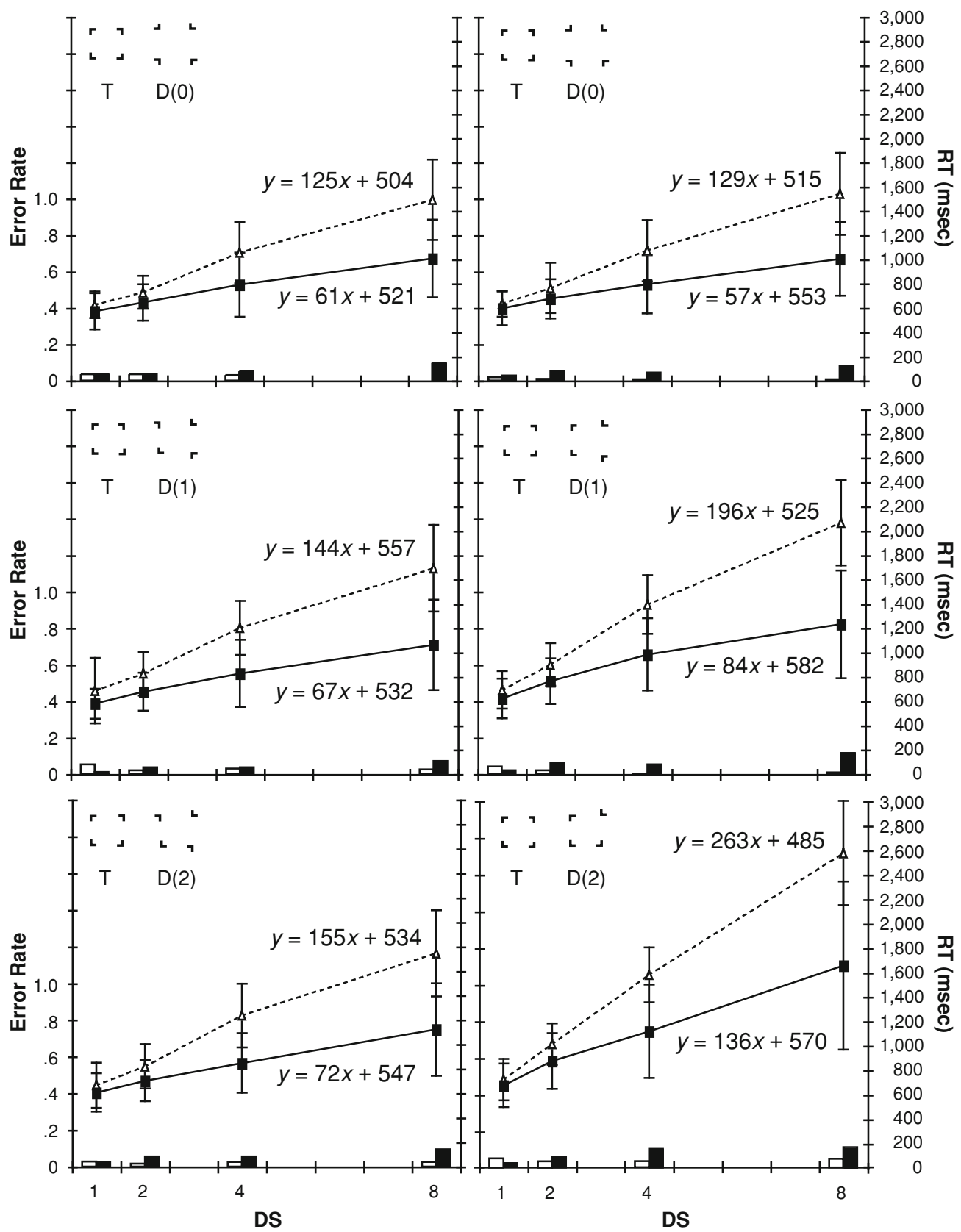

Figure 4. Mean reaction times (RTs, with associated $S D$ s) and error rates in Experiment 2 as a function of display size (DS), separately for open-form (A, left column) and closed-form (B, right column) distractors with zero, one, and two collinear continuations (top, middle, and bottom graphs, respectively). Each graph shows the prototype target $(T)$ and an example of a distractor (D) and plots RTs and error rates separately for target-absent (dotted line, white bars) and target-present (solid line, black bars) trials. In addition, the function for the best-fitting straight line is given for each search RT function, with the search rate and base $R T$ estimates.

were significant; the four-way interaction was not significant. Given the various interactions involving distractor type - in particular, the theoretically interesting distractor type $\times$ display size $\times$ collinear continuation interaction $[F(6,280)=16.32, p<.001]$ - the data sets for open- and closed-form distractor types were analyzed separately by two ANOVAs with the factors of collinear continuations, target, and display size.

For the open-form distractors, the ANOVA revealed that all the main effects $[\mathrm{T}, F(1,7)=71.87, p<.001 ; \mathrm{DS}$, $F(3,21)=18.18, p<.001 ;$ CC, $F(2,14)=12.56, p<$ $.001]$ and two-way interactions were significant $[\mathrm{T} \times \mathrm{DS}$, 
$F(3,119)=89.55, p<.001 ; \mathrm{T} \times \mathrm{CC}, F(2,119)=5.26$, $p<.01 ; \mathrm{DS} \times \mathrm{CC}, F(6,119)=2.23, p<.05]$. In contrast to Experiment 1, the functions relating RT to display size were nonflat, with average search rates of 66.67 and $141.33 \mathrm{msec} /$ item for target-present and -absent trials, respectively. Furthermore, the search time per item increased with an increasing number of collinear continuations in distractors. Although this increase appeared less marked for target-present than for target-absent trials (each additional continuation lengthened the search time per item by 5.5 and $15.0 \mathrm{msec}$, respectively), the three-way interaction was not significant $[F(6,119)=0.62, p=.713]$.

For the closed-form distractors, the ANOVA revealed that all the main effects $[\mathrm{T}, F(1,7)=71.87, p<.001$; DS, $F(3,21)=53.27, p<.001$; CC, $F(2,14)=185.91$, $p<.001]$, the two-way interactions [T $\times \mathrm{DS}, F(3,119)=$ $170.17, p<.001 ; \mathrm{T} \times \mathrm{CC}, F(2,119)=15.07, p<.001$; $\mathrm{DS} \times \mathrm{CC}, F(6,119)=60.64, p<.001]$, and the threeway interaction $[\mathrm{T} \times \mathrm{DS} \times \mathrm{CC}, F(6,119)=4.16, p<$ $.001]$ were significant. As in Experiment 1, an increase in the number of collinear continuations produced an increase in the search time per item, which was significantly less pronounced for target-present than for target-absent trials (each additional continuation lengthened the search time per item by 39.5 and $67.0 \mathrm{msec}$, respectively).

Error analysis. Erroneous responses were relatively rare overall $(4.1 \%$ of all the trials; $5.7 \%$ misses, $2.4 \%$ false alarms). The arcsine-transformed error data were analyzed by means of ANOVAs identical to those applied to the RT data. For the open-form conditions, the ANOVA revealed significant main effects for target and display size $[\mathrm{T}, F(1,7)=10.70, p<.05 ; \mathrm{DS}, F(3,21)=4.02, p<$ $.05]$ and significant interactions of target with display size and with collinear continuations $[\mathrm{T} \times \mathrm{DS}, F(3,119)=$ $15.74, p<.001 ; \mathrm{T} \times \mathrm{CC}, F(2,119)=3.77, p<.05]$. For the closed-form conditions, the ANOVA revealed that all three main effects were significant $[\mathrm{T}, F(1,7)=17.69, p<$ $.01 ; \mathrm{DS}, F(3,21)=4.98, p<.01 ; \mathrm{CC}, F(2,14)=12.12$, $p<.001]$, as well as the target $\times$ display size interaction $[\mathrm{T} \times \mathrm{DS}, F(3,119)=13.74, p<.001]$. In summary, error rates - in particular, miss rates - increased with both display size and the number of collinear continuations (with roughly similar patterns for closed- and open-form conditions). Speed-accuracy trade-offs were not evident.

\section{Discussion}

Reducing the physical specification of the corner junction elements in Experiment 2 resulted in a pronounced decrease in search efficiency, with overall longer search RTs, lengthened search times per item, and higher error rates, in comparison with Experiment 1. However, although search performance was also affected in conditions with open-form distractors (which showed slowed search rates), the detrimental effect of the reduced element specification remained more marked with closedform distractors. In particular, each additional continuation increased the search time per item by $53.25 \mathrm{msec}$ (combined across target-present and -absent trials) in the closed-form condition, but by only $10.25 \mathrm{msec}$ in the open-form condition; these figures compare with 58.25 and $2.75 \mathrm{msec}$, respectively, in Experiment 1. Thus, when the closure of the target figure was reduced as a result of the reduced element specification, the mere presence of collinear continuations in distractors (in the open-form condition) produced interference. However, the interference was significantly increased when the collinear continuations provided cues to figural closure (in the closedform condition).

Note, though, that the interference arising from closure cues appeared slightly less in Experiment 2 than in Experiment 1 (the increase in search time per item with each additional continuation was $53.25 \mathrm{msec}$ in Experiment 2, as compared with $58.25 \mathrm{msec}$ in Experiment 1). This pattern of interference effects suggests that, with the reduced figural "goodness" of the target in Experiment 2, the extent to which the observers were able to use closure cues to guide target detection was reduced, while, at the same time, search performance became more reliant on analyzing collinear continuation cues.

\section{EXPERIMENT 3}

Experiments 1 and 2 provided evidence that variations in the extent of closure in distractors influence the efficiency of target detection. Experiment 1 revealed a search asymmetry, with open-form distractors having only little influence on search efficiency, but with closed-form distractors giving rise to significant interference. When the extent to which closure cues could be used to guide search was reduced overall in Experiment 2, the asymmetrical interference between closed- and open-form distractors was lessened, although not completely abolished. Given this, Experiment 3 was designed to examine the effects of an increase in stimulus closure, in both open- and closedform distractor conditions. In both conditions, stimulus closure was increased by presenting each configuration with a set of four perpendicular lines that terminated adjacent to the unspecified continua between corner junction elements (see Figure 5 for examples), thereby reinforcing these (unspecified) continua.

\section{Method}

The stimulus displays were identical to those in Experiment 1, except that, to each configuration (whether target or distractor), four line segments (each subtending $0.5^{\circ}$ of visual angle) were placed halfway and perpendicular to (i.e., with one end terminating on) the junction-junction continua (see Figure 5A; see Figure 5B for an example display). Eight observers ( 3 of them male, mean age $=$ 25.7 years) with normal or corrected-to-normal visual acuity participated in the experiment. All other details were identical to those in Experiment 1.

\section{Results}

RT analysis. RTs on trials on which a response error was made $(2.1 \%)$ were removed from the data set prior to RT analysis. Figure 6 presents the mean correct RTs and the proportions of error as a function of display size, separately for the open- and closed-form distractors (see figure columns A and B, respectively) and for the various collinear continuation conditions. As can be seen, with the enhanced specification of the junction-junction con- 
A

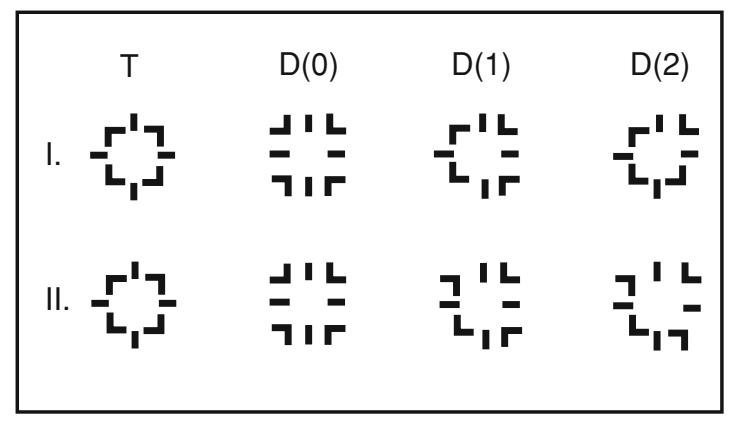

B

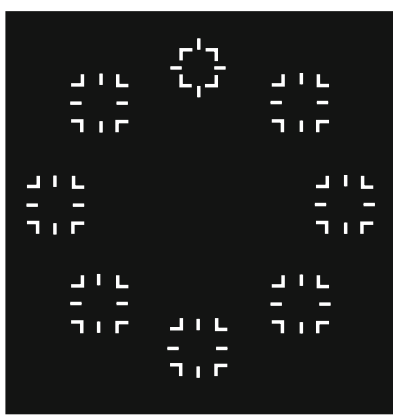

Figure 5. (A) Examples of the target (T) and distractor (D) configurations with additional perpendicular lines halfway between neighboring junction elements in Experiment 3. Distractors could contain zero, one, or two continua $[D(0)-D(2)]$ between adjacent corner junctions that promoted either a closed-form (I) or a corresponding open form (II). (B) Example of a target-present display with eight candidate groupings.

tinua in Experiment 3 (providing closure cues), search was more efficient overall than in Experiment 2, although not quite as efficient as in Experiment 1 (i.e., search rates were comparatively slowed in almost all the conditions, except for the baseline). Importantly, however, relative to Experiment 1, the detrimental effect of the enhanced specification of the junction-junction continua was comparatively greater with open-form than with closed-form distractors.

A four-way ANOVA of the whole data set revealed that all the main effects and all the two- and three-way interactions (except distractor type $\times$ target $\times$ collinear continuations) were significant; the four-way interaction was not significant. Given the various interactions involving distractor type - in particular, the theoretically interesting distractor type $\times$ display size $\times$ collinear continuation interaction $[F(6,280)=3.81, p<.01]$ - the data sets for open- and closed-form distractor types were again analyzed separately by two ANOVAs with the factors of collinear continuations, target, and display size.

For the open-form distractors, the ANOVA revealed that all the main effects [T, $F(1,7)=46.62, p<.001$; DS, $F(3,21)=24.53, p<.001 ;$ CC, $F(2,14)=15.16, p<$ $.001]$ and interactions $[\mathrm{T} \times \mathrm{DS}, F(3,119)=20.41, p<$ $.001 ; \mathrm{T} \times \mathrm{CC}, F(2,119)=8.91, p<.001 ; \mathrm{DS} \times \mathrm{CC}$, $F(6,119)=23.96, p<.001 ; \mathrm{T} \times \mathrm{DS} \times \mathrm{CC}, F(6,119)=$ $3.34, p<.01]$ were significant. In contrast to Experiment 1 , the functions relating RT to display size were nonflat. However, the average search rates, of 33.67 and $78.0 \mathrm{msec} /$ item for target-present and target-absent trials, respectively, were faster than those in Experiment 2 (66.67 and $141.33 \mathrm{msec} /$ item, respectively). Furthermore, the search time per item increased with an increasing number of collinear continuations in distractors, with a differential increase on target-present and -absent trials: 27.5 versus $55.5 \mathrm{msec}$ per collinear continuation (which compares with 5.5 and $15.0 \mathrm{msec}$ in Experiment 2).

For the closed-form distractors, similarly, all the effects were revealed to be significant $[\mathrm{T}, F(1,7)=69.51$, $p<.001 ; \mathrm{DS}, F(3,21)=99.17, p<.001 ; \mathrm{CC}, F(2,14)=$
$224.89, p<.001 ; \mathrm{T} \times \mathrm{DS}, F(3,119)=100.72, p<$ $.001 ; \mathrm{T} \times \mathrm{CC}, F(2,119)=41.26, p<.001 ; \mathrm{DS} \times \mathrm{CC}$, $F(6,119)=115.55, p<.001 ; \mathrm{T} \times \mathrm{DS} \times \mathrm{CC}, F(6,119)=$ 13.31, $p<.001]$. As in Experiments 1 and 2, an increase in the number of collinear continuations produced a very considerable increase in the search time per item (with a differential increase in target-present and -absent trials). However, this deterioration in search performance appeared more marked in Experiment 3 than in both Experiments 1 and 2, at least for the target-present trials: Each additional continuation lengthened the search time per item by $46.0 \mathrm{msec}$ (Experiment 3), as compared with 39.5 and $31.0 \mathrm{msec}$ (Experiments 2 and 1, respectively).

This pattern of increased interference effects in Experiment 3 suggests that the presentation of the perpendicular line segments resulted in stronger closure cues, with both open- and closed-form distractors.

Error analysis. Response errors were relatively rare overall $(2.1 \%$ of all the trials; $2.9 \%$ misses, $1.2 \%$ false alarms). The arcsine-transformed error data were analyzed by means of ANOVAs identical to those applied to the RT data. For the open-form distractors, the ANOVA revealed a significant main effect of target $[\mathrm{T}, F(1,7)=8.02, p<$ $.05]$, due to misses being more frequent than false alarms. For the closed-form distractors, the ANOVA revealed that the main effects of target and collinear continuations [T, $F(1,7)=9.59, p<.05 ; \mathrm{CC}, F(2,14)=9.79, p<.01]$ and the interactions of display size with collinear continuations and with target and collinear continuations [DS $\times$ $\mathrm{CC}, F(6,119)=2.48, p<.05$; $\mathrm{DS} \times \mathrm{T} \times \mathrm{CC}, F(6,119)=$ $2.35, p<.05]$ were significant. Error rates - in particular, miss rates - increased as a function of display size, with the increase being the more pronounced the greater the number of collinear continuations in the distractors. Thus, both RT data and error rates showed similar trends, arguing against speed-accuracy trade-offs.

\section{Discussion}

The addition of perpendicular lines that terminated adjacent to the unspecified continua between corner junctions 
A
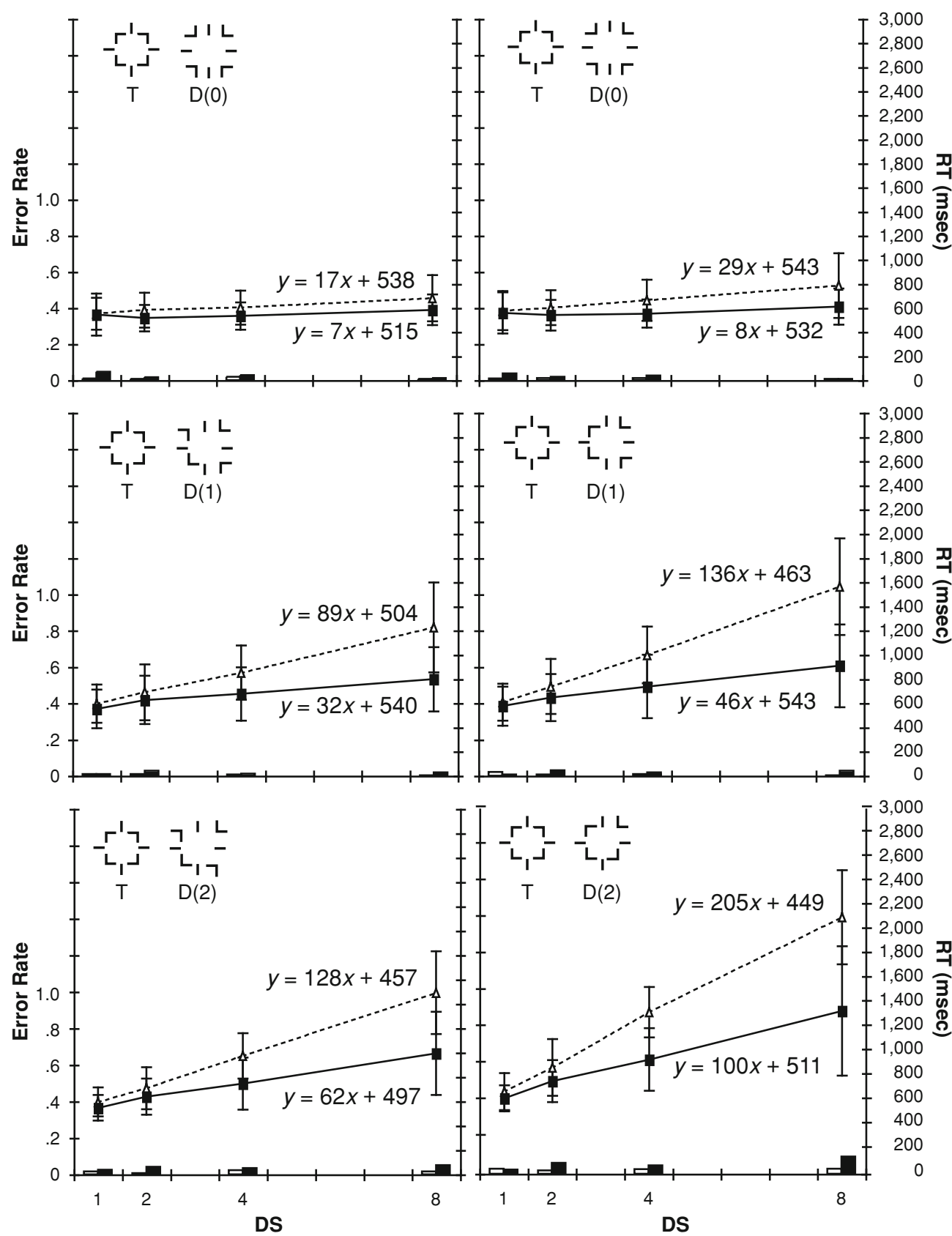

B
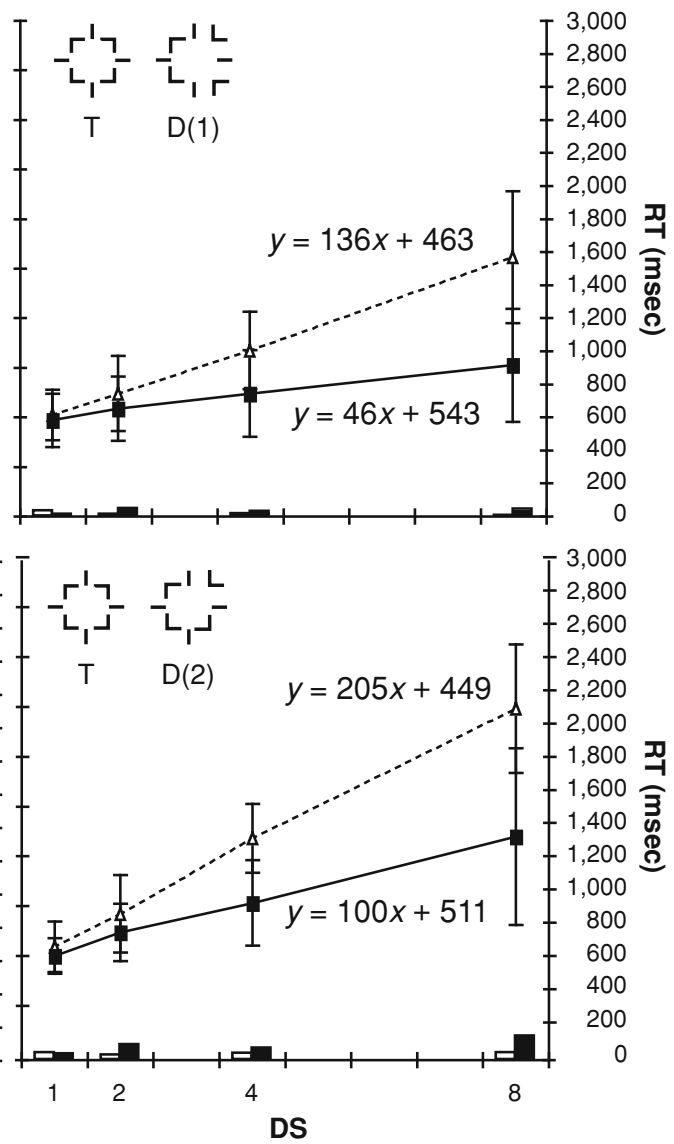

Figure 6. Mean reaction times (RTs, with associated $S D s$ ) and error rates in Experiment 3 as a function of display size (DS), separately for open-form (A, left column) and closed-form (B, right column) distractors with zero, one, and two collinear continuations (top, middle, and bottom graphs, respectively). Each graph shows the prototype target (T) and an example of a distractor (D) and plots RTs and error rates separately for target-absent (dotted line, white bars) and target-present (solid line, black bars) trials. In addition, the function for the best-fitting straight line is given for each search RT function, with the search rate and base $R T$ estimates.

had qualitatively similar effects on search performance with open- and closed-form distractors: In both conditions, the search time per item increased with each additional collinear continuation between corner junctions. In particular, there was a substantial increase even with the open-form distractors (27.5 [55.5] msec per collinear con- tinuation), which was much larger than any effect in the previous experiments (5.5 [15] msec in Experiment 2). Yet this increase was still less marked than that observed with the closed-form distractors (46 [88] msec). Overall, however, the search asymmetry between the open- and the closed-form distractor conditions, established in Experi- 
ment 1, was reduced in Experiment 3, arguably because the presence of the perpendicular lines afforded a greater degree of closure in the open-form, as well as the closedform, distractor condition. Thus, the reduced search asymmetry observed in Experiment 3 provides further evidence in support of the idea that search for closed target forms is influenced by the degree of closure exhibited by the distractors.

\section{EXPERIMENT 4}

Experiments 1-3 provided convergent evidence in support of the proposal that closure has a specific influence on the efficiency of search for collinear, closed target configurations. However, one confounding factor that may have influenced performance in these experiments is global configuration size. This point is best seen when comparing the stimulus configurations used in Experiment 1 (see Figure 2). Here, the target configuration exhibited not only the largest degree of closure, but also the smallest global size - that is, the area encompassed within its four corner junctions. Furthermore, the distractor configurations that interfered least with target detection were generally those that covered a larger area, whereas the (closed-form) distractor configurations, which circumscribed a similar-size area for the target configuration, produced the greatest interference. Consequently, the performance differences observed in Experiment 1 might be better explained in terms of global size variations between the target and the distractor configurations, rather than in terms of variations in the degree of closure.

Although there are arguments against such a size-based account (typically, there is a search asymmetry, so that search is less efficient when the target is smaller than the distractors than when it is larger; see, e.g., Busch \& Müller, 2004), Experiment 4 was designed to examine this alternative account empirically. To do so, in Experiment 4, the size of the distractor configurations was systematically varied, whereas that of the target configuration was kept constant. Relative to the target, the pixels making up the distractors were, on average, more distant from the center of the configuration (target smaller than distractors), at the same average distance (equally large target and distractors), or less distant (target larger than distractors). (The assumption underlying this size manipulation was that global configuration size is computed on the basis of the output of spatiotopically organized detectors that determine the center of gravity of each corner junction; size is then derived by computing the area encompassed by four adjacent points of maximum activity.) Only closed-form distractors were presented in Experiment 4, because these configurations account for the major variation in size and search RTs (see Figure 7A for example target and distractor configurations and Figure 7B for an example display in which the target and the distractors have equal sizes).

\section{Method}

Experiment 4 was identical to the closed-form condition in Experiment 1 , except that size was systematically varied for the distractor configurations. Three size conditions were tested. In the
A
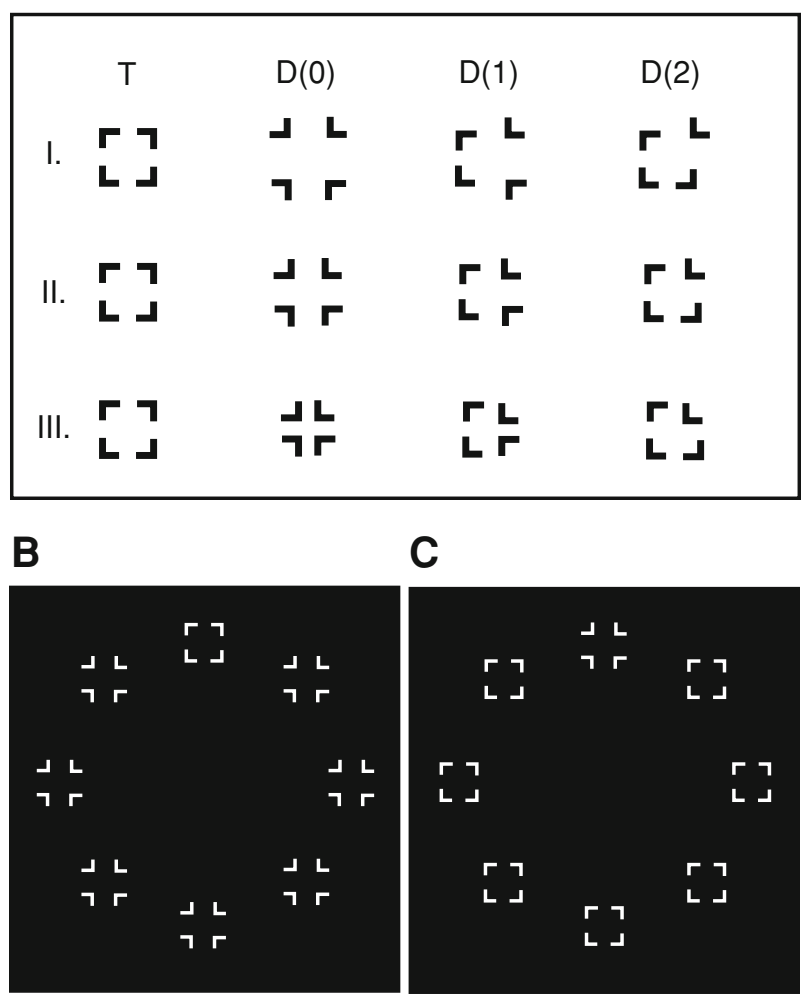

Figure 7. (A) Examples of the target (T) and distractor (D) configurations presented in Experiment 4. Distractors could contain zero, one, or two collinear continuations $[\mathrm{D}(0)-\mathrm{D}(2)]$ between adjacent corner junctions that promoted a closed form. The size of the distractor configurations was varied, so that the target was smaller (I), equally large (II), or larger (III) than the corresponding distractor configurations. (B) Example of a display with eight candidate groupings (corresponding to size variations in A-II) in Experiments 4 and 5. In Experiment 5, the square target condition illustrated in panel $B$ was contrasted with a nonsquare target condition illustrated in panel $\mathbf{C}$.

first, the stimulus configurations were the same as those described in Experiment 1 (target smaller than distractors; see Figure 7A-I). In the second condition, the (average pixel) distance of the corner junctions from the center of each configuration was equal for the target and the distractors (target and distractors equally large; see Figure 7A-II). In the third condition, the target and distractor configurations matched in terms of their outline size (target larger than distractors; see Figure 7A-III). Figure 7B presents an example display for the equally large condition.

Nine observers ( 2 of them male, mean age $=23.7$ years $)$ with normal or corrected-to-normal visual acuity participated in the experiment. The experiment consisted of three sessions, each presenting one size condition, with session order counterbalanced across observers. In summary, the independent variables were size (S; smaller, equal, or larger target), target (T; present or absent), display size (DS; 1, 2, 4, or 8 configurations), and collinear continuations in distractor configurations (CC; 0,1 , or 2 continuations), with 40 trials per condition. All other details were identical to the procedure described in Experiment 1.

\section{Results}

RT analysis. RTs on trials on which a response error was made $(3.5 \%)$ were removed from the data set prior 
to RT analysis. Figure 8 presents the mean correct RTs and the proportions of errors as a function of display size, separately for the smaller-, equal-, and larger-target conditions (see figure columns A, B, and C, respectively) and the various collinear continuation conditions.

The RT data were examined by a repeated measures ANOVA with the factors of size, target, display size, and collinear continuations. There were significant effects for target, display size, and collinear continuations $[\mathrm{T}, F(1,8)=65.41$, $p<.001$; DS, $F(3,24)=59.44, p<.001 ; \mathrm{CC}, F(2,16)=$ $89.45, p<.001 ; \mathrm{T} \times \mathrm{DS}, F(3,504)=102.02, p<.001 ; \mathrm{T}$ $\times \mathrm{CC}, F(2,504)=78.99, p<.001 ; \mathrm{DS} \times \mathrm{CC}, F(6,504)=$
148.16, $p<.001 ; \mathrm{T} \times \mathrm{DS} \times \mathrm{CC}, F(6,504)=26.86, p<$ $.001]$. However, importantly, there were no significant effects involving the factor of size (all $F \mathrm{~s}<2.4$ ). Thus, as in Experiments 1-3, an increase in the specification of closure in distractors gave rise to a slowing of the search rates (significant DS $\times \mathrm{CC}$ and T $\times \mathrm{DS} \times \mathrm{CC}$ interactions; see Figure 8). However, this slowing was not at all influenced by size, indicating that previously noncontrolled variations of the size of the target relative to the distractors is unlikely to account for the observed RT and search rate effects.

Error analysis. Response errors were relatively rare overall $(3.5 \%$ of all the trials; $4.6 \%$ misses, $2.4 \%$ false
A
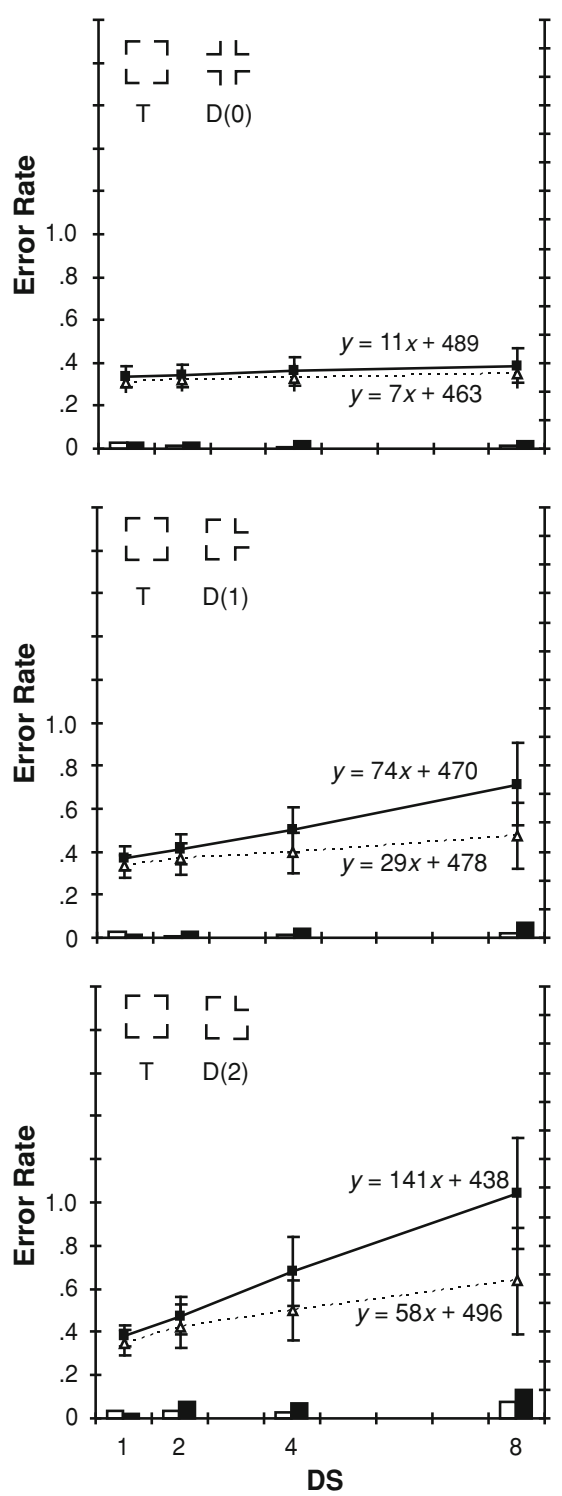

B
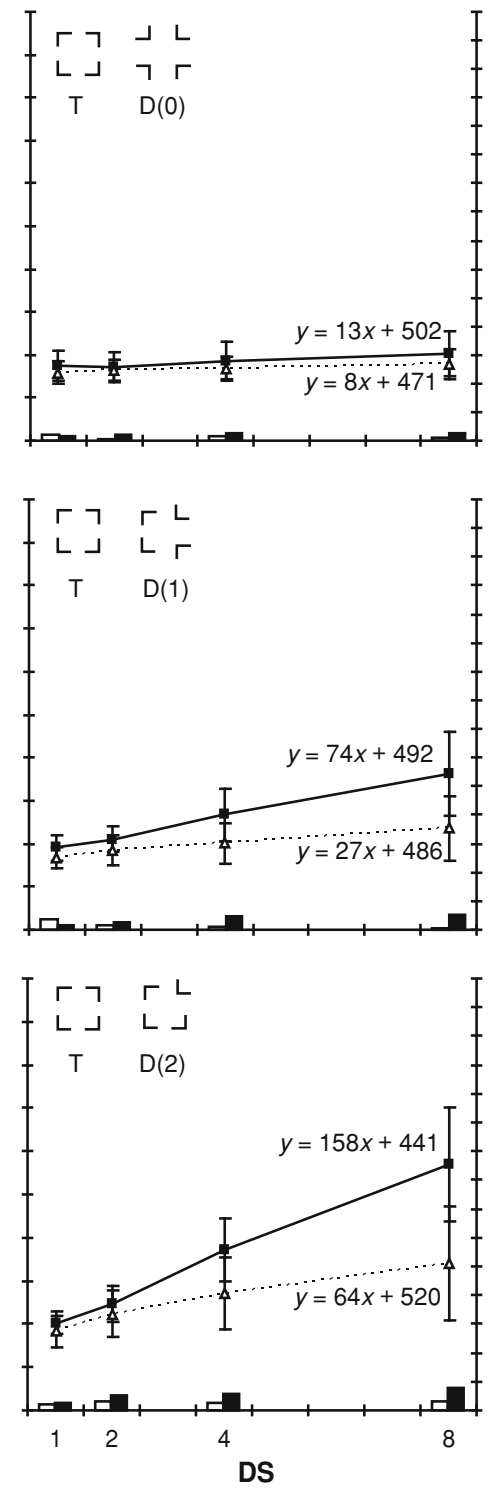

C
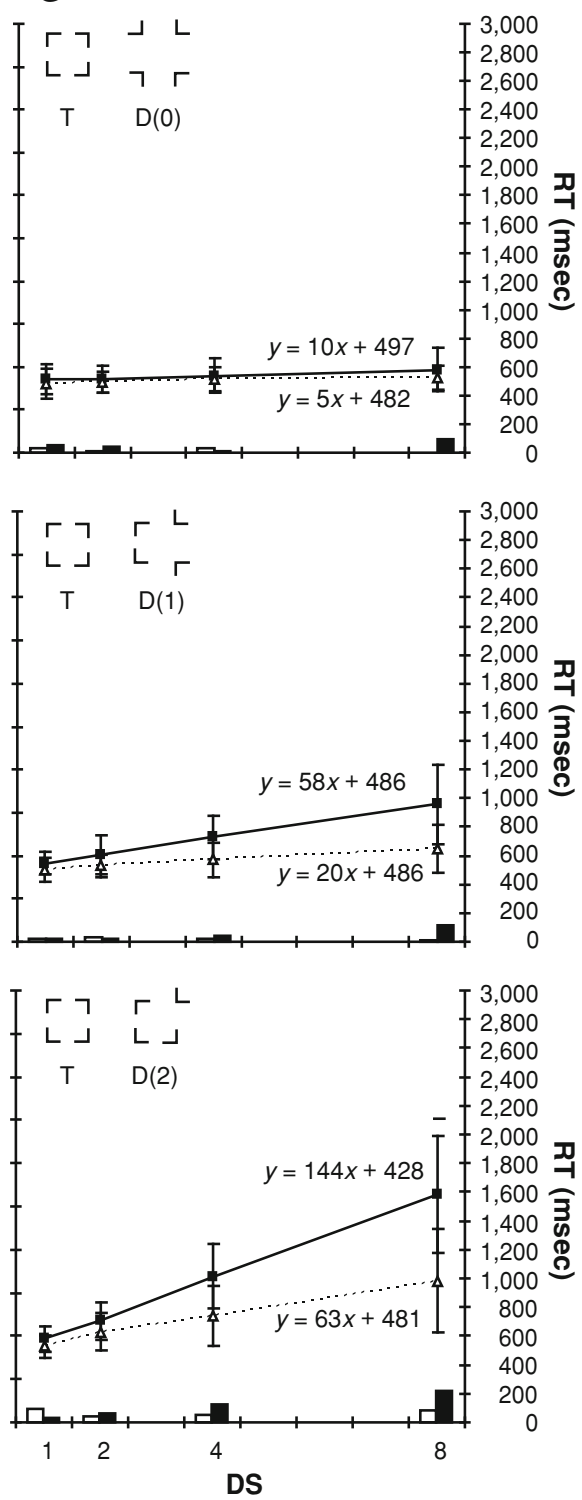

Figure 8. Mean reaction times (RTs, with associated SDs) and error rates in Experiment 4 as a function of display size, separately for targets larger than (A), equally large as (B), and smaller than (C) the corresponding (closed-form) distractors with zero, one, and two collinear continuations (top, middle, and bottom graphs, respectively). Each graph shows the prototype target ( $T$ ) and an example of a distractor (D) and plots RTs and error rates separately for target-absent (solid line, white bars) and target-present (dotted line, black bars) trials. In addition, the function for the best-fitting straight line is given for each search RT function, with the search rate and base RT estimates. 
alarms). The arcsine-transformed error data were analyzed by means of an ANOVA identical to that for the RT data. This analysis revealed significant main effects for target, display size, and collinear continuations [T, $F(1,8)=22.26, p<.01$; DS, $F(3,24)=6.37, p<.01$; $\mathrm{CC}, F(2,16)=12.74, p<.001]$. In addition, target interacted with display size and with the number of collinear continuations $[\mathrm{T} \times \mathrm{DS}, F(3,504)=19.64, p<.001 ; \mathrm{T} \times$ $\mathrm{CC}, F(6,504)=6.49, p<.001]$. Error rates-in particular, miss rates - increased as a function of display size and with an increase in the number of collinear continuations in distractors. However, as with the RT analysis, there were no significant effects resulting from the variation of size. Thus, both RT and error rate data showed similar trends, with no speed-accuracy trade-offs evident.

\section{Discussion}

Variations of size relations between target and distractor configurations did not change the overall pattern of performance, relative to Experiment 1. In particular, there was no evidence of the search asymmetry typically found with size-defined targets (e.g., Busch \& Müller, 2004): Search was not more efficient when the target was larger than the distractors as opposed to when it was smaller. This suggests that target detection was not based on the global size relations between the target and the distractor configurations. However, again, there was a substantial increase in search rates with closed-form distractors, which was independent of size variations (27 [71], 30 [79], and 31.5 [73] msec per collinear continuation for smaller-, equal-, and larger-target conditions, respectively) and was comparable to the effect obtained in Experiment 1 (31 [85.5] msec).

In summary, increasing the specification of closure by means of additional collinear continuations resulted in an equivalent modulation of search performance, as in the previous experiments. However, extending the findings of Experiments 1-3, the results of Experiment 4 ruled out the possibility that this modulation is explicable in terms of (potentially confounding) size relations between the target and the distractor configurations.

\section{EXPERIMENT 5}

However, even if a size confound can be ruled out (Experiment 4), another explanation for the pattern of interference effects revealed with closed-form (as compared with open-form) distractors in Experiments 1-4 is in terms of the similarity of junction element orientations between the target and the distractor configurations. For example, according to the contrast model of Tversky (1977; see also Edelman, 1998, and Duncan \& Humphreys's, 1989, similarity theory of visual search), search efficiency varies as a function of the similarity between configurations that share distinctive features: Similarity is the greater, the greater the number of common features. Applied to the present results, variations in search efficiency might arise because closed-form distractors, which produce the largest interference with target detection, share a greater number of (inward-oriented) junctions with the target configu- ration, relative to open-form distractors, which produce the least, if any, interference. Thus, on this account, interference would arise merely as a result of feature overlap between target and distractor configurations; that is, there is no special role for grouping (the computation of interelement relations) - in particular, grouping by closure.

Note that the results of the previous experiments rule out a simple similarity account, with similarity defined in terms of the match between target and distractor junction feature lists, without regard to junction orientation direction and/or junction location relative to a target description (template): On this account, the baseline distractor configurations should have produced the greatest interference, because there is a perfect match in the feature lists (all the junctions present in the target are also present in the distractors). Thus, the only account that would work is one in which similarity is computed on the basis of feature matches that take junction orientation and/or location with respect to the target template into consideration. That is, any viable measure of similarity is relational, in the sense that feature matches must be counted with respect to a superordinate target description. This does not mean, however, that the features are grouped in any sense (even though most current accounts would assume that templatebased, or top-down, biasing of target-defining features would promote feature-"object" grouping at lower levels of the system; see, e.g., Desimone \& Duncan, 1995; Duncan \& Humphreys, 1989). That is, the critical assumption of the similarity account is that grouping based on collinearity and closure cues is not involved in determining search performance.

The open-form results of Experiments 2 and 3 are inconsistent with this assumption. In both experiments, there was an increase in the search time per item when the number of collinear continuations increased, even though the number of corner junctions that exhibited a mismatch with the target description was the same (namely, three), whether distractors contained one or two collinear continuations. This would argue in favor of, at least, collinearitybased grouping's playing a role.

Nevertheless, Experiment 5 was designed to test the similarity account further by evaluating the effect of closure independently of junction orientation matches (with reference to a target description). This was done by contrasting two search conditions, in both of which the target and the distractor configurations were maximally dissimilar. One condition was the same as the baseline condition in the previous experiments: The target was a closed square, and the distractors were nonclosed crosses (see Figure 7B). In the other condition, the situation was reversed: The target was a nonclosed cross, and the distractors were closed squares (see Figure 7C). The similaritybased account would predict no differences between these two conditions: Search should be equally efficient regardless of which configuration is assigned the role of the target and which that of the distractor. In contrast, if the system is biased to compute closure cues in parallel (but not cues to nonclosure), then, according to Treisman and Gormican (1988), one could expect a search asymmetry, so that closed square targets are detected efficiently 
among nonclosed cross distractors, whereas nonclosed cross targets are detected relatively inefficiently among closed square distractors.

\section{Method}

Experiment 5 was basically identical to Experiment 1, except that only two possible configurations were employed, both serving either as the target or as distractors. In one condition, the observers were required to search for a collinear target square among nonsquare distractor configurations (see Figure 7B for an example display). In a second condition, the nonsquare configuration served as the target, and the square configuration as distractors (see Figure $7 \mathrm{C}$ for an example display). To rule out effects of size variations, target and distractor groupings were presented with junctions at equal (average pixel) distances from the center of a configuration (see Experiment 4, Figure 7A-II). Sixteen observers (4 of them male, mean age $=27.5$ years) with normal or corrected-to-normal visual acuity participated in the experiment. To rule out order effects of the target type (square or nonsquare), the observers were randomly assigned to one of two experimental groups, one searching for a square target (among nonsquare distractors) and the other searching for a nonsquare target (among square distractors). The experiment consisted of one session with four blocks of 80 trials each. In summary, the independent variables were target ( $T$; present or absent), display size (DS; 1, 2, 4, or 8 configurations), and target type (TT; square or nonsquare), with 40 trials per experimental condition. All other details were identical to the procedure described for Experiment 1.

\section{Results}

RT analysis. RTs on trials on which a response error was made (3.3\%) were removed prior to analysis. Figure 9 presents the mean correct RTs and the error rates as a function of display size for square (panel A) and nonsquare (panel B) target conditions. In a first analysis step, RTs in square and nonsquare target conditions were compared by means of a $t$ test, which revealed a robust difference between the two conditions $[t(14)=3.12, p<.01]$ : RTs were $137 \mathrm{msec}$ shorter, on average, for square than for nonsquare targets.
Next, RTs were compared in a mixed-design ANOVA, with the within-subjects factors of target and display size and the between-subjects factor of target type. This ANOVA revealed that the main effects of target and display size and their interaction were significant $[\mathrm{T}$, $F(1,14)=29.57, p<.001 ; \mathrm{DS}, F(3,42)=23.11, p<$ $.001 ; \mathrm{T} \times \mathrm{DS}, F(3,42)=10.56, p<.001]$. Of greater theoretical importance, display size interacted significantly with target type, and the three-way interaction was borderline significant [DS $\times$ TT, $F(1,14)=9.12, p<$ $.001 ; \mathrm{T} \times \mathrm{DS} \times \mathrm{TT}, F(3,42)=2.68, p=.059]$. RTs were longer and the search rates increased when the nonsquare configuration served as the target, as compared with the square target condition. Thus, there is a clear performance asymmetry between targets defined as square and nonsquare configurations, with search for the latter being less efficient overall.

Error analysis. Response errors were relatively rare overall (3.3\% of all the trials) and did not differ between square and nonsquare target conditions. An ANOVA of the arcsine-transformed error data revealed only a significant main effect for target [T, $F(1,14)=5.59, p<.05]$, with $3.9 \%$ misses versus $2.7 \%$ false alarms.

\section{Discussion}

Experiment 5 was designed to evaluate whether the proposed effect of closure on search could be attributed to the similarity in terms of individual corner junction orientations shared by target and distractor configurations. The results revealed a clear effect of closure: The search rates were approximately four times slower for nonsquare targets among square distractors $(23[36] \mathrm{msec})$ than for square targets among nonsquare distractors (4 [9] msec), and performance in the latter case was similar to that in the baseline condition in Experiment 1 (3 [7] msec). An account based on the computation of element similarities
A

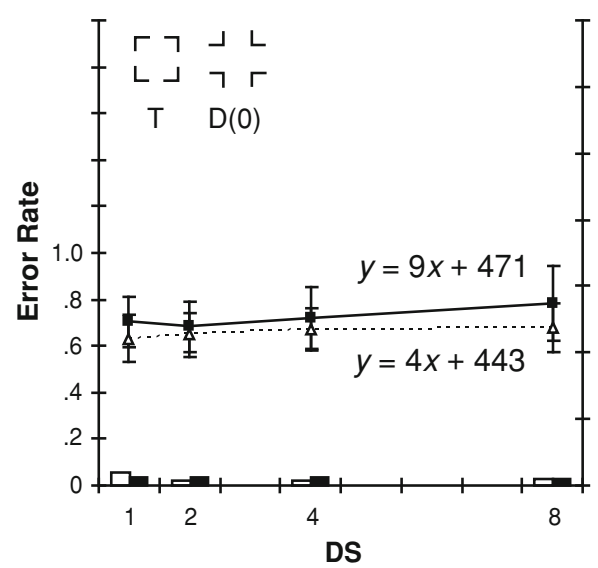

B

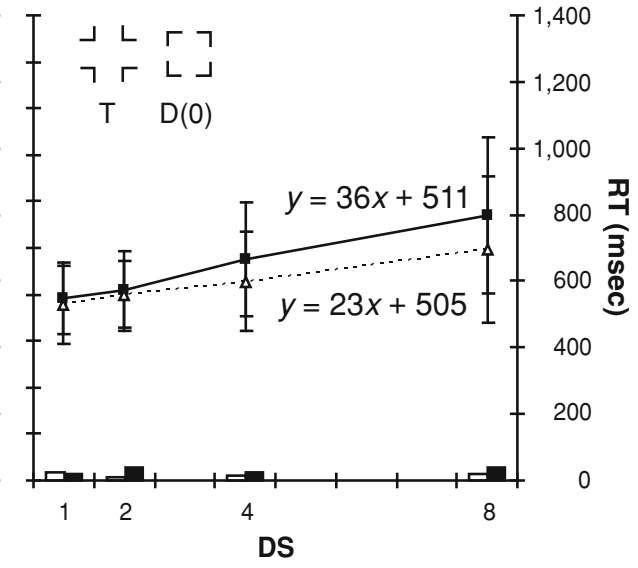

Figure 9. Mean reaction times (RTs, with associated $S D s$ ) and error rates in Experiment 5 as a function of display size, separately for square (A) and nonsquare (B) target conditions. Each graph shows the prototype target (T) and an example of a distractor (D) and plots RTs and error rates separately for target-absent (solid line, white bars) and target-present (dotted line, black bars) trials. In addition, the function for the best-fitting straight line is given for each search RT function, with the search rate and base $R T$ estimates. 
between target and distractor configurations cannot easily explain this search asymmetry. Rather, the differential search efficiency between the two conditions is likely to result from grouping by means of closure, resulting in efficient detection of a square target (possessing closure) and inefficient detection of a nonsquare target (where the distractors, but not the target, possess closure).

\section{GENERAL DISCUSSION}

The results of Experiments 1-5 provide convergent evidence that search for a collinear target configuration is influenced by closure. In Experiment 1, we examined the effects of open- versus closed-form distractors on detection of a target composed of collinearly arranged corner junction elements. The results suggested that distractors interfere with target detection on the basis of closure (see Table 1, columns 2 and 3). In Experiments 2 and 3, we investigated the effects of a reduction and an amplification, respectively, on the potential for closure. In Experiment 2, the length of the line segments making up the corner junctions in target and distractor configurations was reduced. This was meant to lead to a reduction of closure afforded by collinear line segments and, consequently, a reduction of closure-based interference. The results were as expected. Although search was overall less efficient in Experiment 2 than in Experiment 1, the performance differences between the closed- and the open-form distractor conditions were reduced, suggesting that the observers were less reliant on closure to guide target detection (see Table 1, columns 4 and 5). In Experiment 3, the target and distractor configurations were presented with the addition of line segments positioned halfway between and terminating on the junction-junction continua, which were meant to enhance closure cues. This led to greater interference, as was expected. Recall that the interference was increased (relative to the previous experiments) not only with closed-, but also with open-form distractors (see Table 1, columns 6 and 7), arguably due to the added closure cues provided by the perpendicular line terminations.

Experiment 4 was designed to rule out the possibility that the effects attributed to closure simply arose due to systematic differences in global size between the target and the distractor configurations. However, the results of Experiment 4 (in which the global size of the distractor configurations was varied) mirrored those of Experiment 1, with, at most, minor contributions attributable to global size differences.

Table 1

Search Rate Increases (in Milliseconds/Item) per Collinear Continuation in Open- and Closed-Form Distractors for Experiments 1-3

\begin{tabular}{lcccccccc}
\hline & \multicolumn{2}{c}{ Experiment 1 } & & \multicolumn{2}{c}{ Experiment 2 } & & \multicolumn{2}{c}{ Experiment 3 } \\
\cline { 2 - 3 } \cline { 8 - 9 } & Open & Closed & & Open & Closed & & Open & Closed \\
\hline TA & 4.0 & 85.5 & & 15.0 & 67.0 & & 55.5 & 88.0 \\
TP & 1.5 & 31.0 & & 5.5 & 39.5 & & 27.5 & 46.0 \\
Mean & 2.3 & 58.3 & & 10.3 & 53.3 & & 41.5 & 67.0 \\
\hline
\end{tabular}

Note-Increases were estimated as $[\mathrm{D}(2)-\mathrm{D}(0)] / 2$, where $\mathrm{D}(n)$ is the search rate for a given distractor $\mathrm{D}$ with $n$ collinear continuations. TA, target absent; TP, target present.
Finally, Experiment 5 showed that similarities between the orientations of the corner junctions (with reference to a target description) alone cannot explain the interference effects revealed in the previous experiments. Search for a closed square target among nonclosed cross distractors was found to be efficient, whereas search for a nonclosed cross target among closed square distractors was comparatively inefficient. This indicates that the system is biased to group neighboring elements by means of closure, which influences search performance (it expedites search when the target is uniquely defined by closure, and it slows search when the distractors, but not the target, exhibit closure).

Thus, taken together, an account assuming closure-based grouping (which, in turn, assumes collinearity-based grouping) offers the most coherent and parsimonious explanation of the interference effects observed in the present study. Although contributions by global-size differences between target and distractor configurations and mere similarity in terms of junction orientations (with reference to a target description) cannot be ruled out, the latter are relatively minor and not entirely consistent with the effects observed.

The proposal of shape extraction on the basis of grouping by closure (as the best account of the present findings) is in close agreement with previous reports. Evidence from texture discrimination tasks (Elder \& Zucker, 1993; Field et al., 1993; Kovács \& Julesz, 1993) shows that closure of boundary contours may be exploited prior to textural analysis in order to segregate regions in 2-D space. In addition, evidence from visual search has been interpreted in terms of perceptual groups, rather than individual items, forming the basis of attentional selection (Duncan, 1984; Wang, Kristjansson, \& Nakayama, 2005). In agreement with this possible role of perceptual organization for search, several reports have shown that shapes may be combined in parallel to aid target detection (e.g., Donnelly et al., 1991; Donnelly et al., 1998; Treisman \& Paterson, 1984). Complementary to these findings, the results presented here indicate that closure plays a primary role, guiding search for configural targets.

The effects of closure described above also suggest that the processes that link individual elements into groups are relatively complex. Classical Gestalt-psychological approaches (e.g., Wertheimer, 1923) imply that closure operates as a binary property, with groupings either possessing or not possessing closure as a salient attribute. By contrast, the present findings argue in favor of closure's being a graded property of objects in visual search. In particular, the physical element specification, the number of collinear junction-junction continuations, and the inward orientation of the corner junctions influenced search performance, suggesting that the closure-based grouping of elements into more global configurations operates in a graded fashion, with a number of computational steps in element integration. Thus, closure may be taken to represent a property that is derived in a complex geometric extraction process. A consequence of this extraction of closure is an increase in sensitivity for the detection of forms that exhibit this property (Kovács \& Julesz, 1993) or, in analogy to closure, objects that possess topological features, such as holes (Chen, 1982, 2005; see also Pomerantz, 2003). 
Consequently, closure may be regarded as a graded property of groupings, enhancing the saliency of a given configuration. A corresponding selection process on the basis of closure could be implemented in the visual system in terms of feedback connections between different levels of cortical organization. In this framework, closure would be detected at a relatively high level of processing, with recurrent connections supporting the extraction of grouping operations between neighboring junctions at lower levels only subsequently (Hochstein \& Ahissar, 2002; Roelfsema, Lamme, Spekreijse, \& Bosch, 2002; see also Di Lollo, Enns, \& Rensink, 2000).

Possible mechanisms for bringing about element integration may employ principles of interpolation (Kellman $\&$ Shipley, 1991) and extrapolation (Shipley \& Kellman, 2003). According to object interpolation approaches, early stages in visual processing establish links between orientation-selective units via lateral interactions to group neighboring elements in the field (e.g., Grossberg, Mingolla, \& Ross, 1997). As a result of this interpolation process, contours are formed in regions without physical correspondence. Form integration may, in addition, be based on extrapolation, which constitutes a weaker form of boundary integration. Extrapolated contours do not, by themselves, generate contours between physical elements but can strengthen the percept of a given configuration toward one possible interpretation. To explain the present results, inter- and extrapolation could represent possible mechanisms that link the individual fragments into groups. If a closed form results from interpolation- and extrapolation-based integration processes, its saliency for search is enhanced. Consequently, closure would provide valuable additional information that the visual system can exploit in order to achieve efficient target detection.

Computational models of shape extraction support the evidence presented in the present study. In particular, the special role of closed forms in search may be explained in terms of the concept of salient regions (Stanley \& Rubin, 2003). Salient regions can form basic image descriptors generated to rapidly guide selective visual processing to candidate target configurations (in extension of featurebased salience in search; see Itti \& Koch, 2003, for a review). Within this framework, closure may be regarded as one specific property aiding region segregation, in order to mark particular regions, or groupings, for prioritized attentional processing.

\section{AUTHOR NOTE}

This work was supported by German Research Foundation (DFG) Project Grants MU 1564/2 and EL 248/2 to H.J.M. and M.A.E., respectively. We thank Pilar Gamez-Moreno and Ayala Strulson for help with running the experiments and Jim Enns, James R. Pomerantz, and two anonymous reviewers for valuable comments on an earlier draft of the manuscript. Correspondence concerning this article should be addressed to M. Conci, Allgemeine und Experimentelle Psychologie, Department Psychologie, Ludwig-Maximilians Universität, Leopoldstr. 13, D-80802 Munich, Germany (e-mail: conci@psy.uni-muenchen.de).

\section{REFERENCES}

Busch, A., \& MüLler, H. J. (2004). The Ebbinghaus illusion modulates visual search for size-defined targets: Evidence for preattentive processing of apparent object size. Perception \& Psychophysics, 66, 475-495.

CHEN, L. (1982). Topological structure in visual perception. Science, 218, 699-700.

CHEN, L. (2005). The topological approach to perceptual organization. Visual Cognition, 12, 553-585.

CONCI, M., MülleR, H. J., \& ElLiott, M. A. (2007). The contrasting impacts of global and local object attributes on Kanizsa figure detection. Manuscript submitted for publication.

Davis, G., \& Driver, J. (1994). Parallel detection of Kanizsa subjective figures in the human visual system. Nature, 371, 291-293.

Desimone, R., \& Duncan, J. (1995). Neural mechanisms of selective visual attention. Annual Review of Neuroscience, 18, 193-222.

Di Lollo, V., Enns, J. T., \& RENSINK, R. A. (2000). Competition for consciousness among visual events: The psychophysics of reentrant visual processes. Journal of Experimental Psychology: General, 129, 481-507.

Donnelly, N., HumPhreys, G. W., \& RidDOCH, M. J. (1991). Parallel computation of primitive shape descriptions. Journal of Experimental Psychology: Human Perception \& Performance, 17, 561-570.

Donnelly, N., WeEKes, B., HumphreYs, G. W., \& AlbON, A. (1998). Processes involved in the computation of a shape description. Journal of Experimental Psychology: Human Perception \& Performance, 24, 1119-1130.

DUNCAN, J. (1984). Selective attention and the organization of visual information. Journal of Experimental Psychology: General, 113, 501-517.

DüCAN, J., \& HuMPhreys, G. W. (1989). Visual search and stimulus similarity. Psychological Review, 96, 433-458.

Edelman, S. (1998). Representation is representation of similarities. Behavioral \& Brain Sciences, 21, 449-498.

ELDER, J., \& ZUCKER, S. (1993). The effect of contour closure on the rapid discrimination of two-dimensional shapes. Vision Research, 33, 981-991.

FiELD, D. F., HAYES, A., \& Hess, R. F. (1993). Contour integration by the human visual system: Evidence for a local "association field." Vision Research, 33, 173-193.

FoUND, A., \& MüLLER, H. J. (1997). Local and global orientation in visual search. Perception \& Psychophysics, 59, 941-963.

Gegenfurtner, K. R., Brown, J. E., \& Rieger, J. (1997). Interpolation processes in the perception of real and illusory contours. Perception, 26, $1445-1458$.

GeISLER, W. S., \& Super, B. J. (2000). Perceptual organization of twodimensional patterns. Psychological Review, 107, 677-708.

GraboweCKy, M., \& TreISMAN, A. M. (1989). Attention and fixation in subjective contour perception. Investigative Ophthalmology \& Visual Science, 30, 457.

Grossberg, S., Mingolla, E., \& Ross, W. D. (1997). Visual brain and visual perception: How does the cortex do perceptual grouping? Trends in Neurosciences, 20, 106-111.

Gurnsey, R., PoIRIER, F. J. A. M., \& GASCon, E. (1996). There is no evidence that Kanizsa-type subjective contours can be detected in parallel. Perception, 25, 861-874.

Han, S., Humphreys, G. W., \& Chen, L. (1999a). Parallel and competitive processes in hierarchical analysis: Perceptual grouping and encoding of closure. Journal of Experimental Psychology: Human Perception \& Performance, 25, 1411-1432.

HAN, S., Humphreys, G. W., \& CHEN, L. (1999b). Uniform connectedness and classical Gestalt principles of perceptual grouping. Perception \& Psychophysics, 61, 661-674

HochSTEIN, S., \& AHISSAR, M. (2002). View from the top: Hierarchies and reverse hierarchies in the visual system. Neuron, 36, 791-804.

Humphreys, G. W., Quinlan, P. T., \& Riddoch, M. J. (1989). Grouping processes in visual search: Effects with single- and combined-feature targets. Journal of Experimental Psychology: General, 118, 258-279.

IтTI, L., \& KоCH, C. (2003). Computational modelling of visual attention. Nature Reviews Neuroscience, 2, 194-203.

KANIZSA, G. (1955). Margini quasi-percettivi in campi con stimolazione omogenea. Rivista di Psicologia, 49, 7-30.

Kellman, T. F., \& Shipley, P. J. (1991). A theory of interpolation in object perception. Cognitive Psychology, 23, 141-221.

KoffKa, K. (1935). Principles of Gestalt psychology. New York: Harcourt.

Kovács, I., \& Julesz, B. (1993). A closed curve is much more than 
an incomplete one: Effect of closure in figure-ground segmentation. Proceedings of the National Academy of Sciences, 90, 7495-7497.

Moore, C. M., \& EgETH, H. (1997). Perception without attention: Evidence for grouping under conditions of inattention. Journal of Experimental Psychology: Human Perception \& Performance, 23, 339-352.

PoMERANTZ, J. R. (2003). Wholes, holes and basic features in vision. Trends in Cognitive Sciences, 7, 471-473.

Pomerantz, J. R., Sager, L. C., \& Stoever, R. J. (1977). Perception of wholes and of their component parts: Some configural superiority effects. Journal of Experimental Psychology: Human Perception \& Performance, 3, 422-435.

RENSINK, R. A., \& ENNS, J. T. (1995). Preemption effects in visual search: Evidence for low-level grouping. Psychological Review, 102, 101-130.

Roelfsema, P. R., Lamme, V. A. F., Spekreisse, H., \& BosCh, H. (2002). Figure-ground segregation in a recurrent network architecture. Journal of Cognitive Neuroscience, 14, 525-537.

ShIPLEY, T. F., \& Kellman, P. J. (1992). Strength of visual interpolation depends on the ratio of physically specified to total edge length. Perception \& Psychophysics, 52, 97-106.

Shipley, T. F., \& Kellman, P. J. (2003). Boundary completion in illusory contours: Interpolation or extrapolation? Perception, 32, 985-999.

Stanley, D. A., \& Rubin, N. (2003). fMR activation in response to illusory contours and salient regions in the human lateral occipital complex. Neuron, 37, 323-331.

Treisman, A. M., \& Gelade, G. (1980). A feature-integration theory of attention. Cognitive Psychology, 12, 97-136.

Treisman, A. [M.], \& Gormican, S. (1988). Feature analysis in early vision: Evidence from search asymmetries. Psychological Review, 95, $15-48$
Treisman, A. [M.], \& Paterson, R. (1984). Emergent features, attention, and object perception. Journal of Experimental Psychology: Human Perception \& Performance, 10, 12-31.

TVERSKY, A. (1977). Features of similarity. Psychological Review, 84, 327-352.

WANG, D., Kristuansson, A., \& NaKayama, K. (2005). Efficient visual search without top-down or bottom-up guidance. Perception \& Psychophysics, 67, 239-253.

WERTHEIMER, M. (1923). Untersuchungen zur Lehre von der Gestalt. Psychologische Forschung, 4, 301-350.

Wolfe, J. M. (1998). What can 1 million trials tell us about visual search? Psychological Science, 9, 33-39.

Wolfe, J. M., \& Horowitz, T. S. (2004). What attributes guide the deployment of visual attention and how do they do it? Nature Reviews Neuroscience, 5, 1-7.

\section{NOTE}

1. With Figure 1B, some observers report an illusory diamond lying in front of the illusory square. However, this impression is much weaker than that of the illusory square in Figure 1A; furthermore, unlike the illusory square, this form is not perceived with short presentation times (see Gegenfurtner, Brown, \& Rieger, 1997).

(Manuscript received January 10, 2005; revision accepted for publication February 24, 2006.) 